\title{
Pach's selection theorem does not admit a topological extension
}

\author{
Imre Bárány* $\quad$ Roy Meshulam ${ }^{\dagger} \quad$ Eran Nevo $^{\ddagger} \quad$ Martin Tancer $^{\S}$
}

September 18, 2018

\begin{abstract}
Let $U_{1}, \ldots, U_{d+1}$ be $n$-element sets in $\mathbb{R}^{d}$. Pach's selection theorem says that there exist subsets $Z_{1} \subset U_{1}, \ldots, Z_{d+1} \subset U_{d+1}$ and a point $u \in \mathbb{R}^{d}$ such that each $\left|Z_{i}\right| \geq c_{1}(d) n$ and $u \in \operatorname{conv}\left\{z_{1}, \ldots, z_{d+1}\right\}$ for every choice of $z_{1} \in Z_{1}, \ldots, z_{d+1} \in Z_{d+1}$. Here we show that this theorem does not admit a topological extension with linear size sets $Z_{i}$. However, there is a topological extension where each $\left|Z_{i}\right|$ is of order $(\log n)^{1 / d}$.
\end{abstract}

\section{Introduction}

Pach's homogeneous selection theorem is the following key result in discrete geometry.

Theorem 1.1 (Pach [12]). For $d \geq 1$ there exists a constant $c_{1}(d)>0$ such that the following holds. For any $n$-element sets $U_{1}, \ldots, U_{d+1}$ in $\mathbb{R}^{d}$, there exist subsets $Z_{1} \subset U_{1}, \ldots, Z_{d+1} \subset$ $U_{d+1}$ and a point $u \in \mathbb{R}^{d}$ such that each $\left|Z_{i}\right| \geq c_{1}(d) n$ and $u \in \operatorname{conv}\left\{z_{1}, \ldots, z_{d+1}\right\}$ for every choice of $z_{1} \in Z_{1}, \ldots, z_{d+1} \in Z_{d+1}$.

This result was proved by Bárány, Füredi, and Lovász [3] for $d=2$ and by Pach [12] for general $d$. Here we show that this theorem does not admit a topological extension when the size of the $Z_{i}$ is linear in $n$, but does admit one when the sizes are of order $(\log n)^{1 / d}$. Now we reformulate Theorem 1.1 and then we state the topological extension.

Throughout the paper we will identify an abstract simplicial complex $X$ with its geometric realization. For $k \geq 0$, let $X^{(k)}$ denote the $k$-dimensional skeleton of $X$ and let $X(k)$ be the family of $k$-dimensional faces of $X$. For an abstract simplex $\sigma=\left\{v_{0}, \ldots, v_{k}\right\} \in X(k)$, we write $\left\langle v_{0}, \ldots, v_{k}\right\rangle$ for its geometric realization.

Let $\Delta_{n-1}$ denote the $(n-1)$-simplex. Consider $d+1$ sets $V_{1}, \ldots, V_{d+1}$, each of size $n$, and their join

$$
\left(\Delta_{n-1}^{(0)}\right)^{*(d+1)} \cong V_{1} * \cdots * V_{d+1}:=\left\{\sigma \subset \bigcup_{i=1}^{d+1} V_{i}:\left|\sigma \cap V_{i}\right| \leq 1 \text { for all } 1 \leq i \leq d+1\right\} .
$$

\footnotetext{
${ }^{*}$ Rényi Institute, Hungarian Academy of Sciences, POB 127, 1364 Budapest, Hungary and Department of Mathematics, University College London, Gower Street, London, WC1E 6BT, UK. email: barany@renyi.hu

${ }^{\dagger}$ Department of Mathematics, Technion - Israel Institute of Technology, Haifa 32000, Israel. email: meshulam@math.technion.ac.il

${ }^{\ddagger}$ Einstein Institute of Mathematics, The Hebrew University of Jerusalem, Jerusalem 91904, Israel. email: nevo@math.huji.ac.il

${ }^{\S}$ Department of Applied Mathematics, Charles University in Prague, Malostranské náměstí 25, 11800 , Praha 1, Czech Republic. email: tancer@kam.mff.cuni.cz
} 
Trivially, there is an affine map $f:\left(\Delta_{n-1}^{(0)}\right)^{*(d+1)} \rightarrow \mathbb{R}^{d}$ that is a bijection between $V_{i}$ and $U_{i}$ for each $i$ (where $U_{i}$ are the sets from the statement of Pach's theorem). In this setting the homogeneous selection theorem says that there exist subsets $Z_{i} \subset V_{i}$ such that $\left|Z_{i}\right| \geq c_{1}(d) n$ and

$$
\bigcap_{z_{1} \in Z_{1}, \ldots, z_{d+1} \in Z_{d+1}} f\left(\left\langle z_{1}, \ldots, z_{d+1}\right\rangle\right) \neq \emptyset
$$

Assume now that $f$ is not affine but only continuous. For a mapping $f:\left(\Delta_{n-1}^{(0)}\right)^{*(d+1)} \rightarrow$ $\mathbb{R}^{d}$, let $\tau(f)$ denote the maximal $m$ such that there exist $m$-element subsets $Z_{1} \subset V_{1}, \ldots, Z_{d+1} \subset$ $V_{d+1}$ that satisfy

$$
\bigcap_{z_{1} \in Z_{1}, \ldots, z_{d+1} \in Z_{d+1}} f\left(\left\langle z_{1}, \ldots, z_{d+1}\right\rangle\right) \neq \emptyset .
$$

Define the topological Pach number $\tau(d, n)$ to be the minimum of $\tau(f)$ as $f$ ranges over all continuous maps from $\left(\Delta_{n-1}^{(0)}\right)^{*(d+1)}$ to $\mathbb{R}^{d}$. Our main result is the following:

Theorem 1.2. For $d \geq 1$ there exists a constant $c_{2}(d)=O(d)$ such that $\tau(d, n) \leq c_{2}(d) n^{1 / d}$ for all $n \geq(2 d)^{d}$.

For a lower bound on $\tau(d, n)$ we only have the following:

Theorem 1.3. For $d \geq 1$ there exists a constant $c_{3}(d)>0$ such that $\tau(d, n) \geq c_{3}(d)(\log n)^{1 / d}$ for all $n$.

Motivation and background. Theorem 1.1 is a descendant of the following selection theorem.

Theorem 1.4 (First selection theorem). Let $P$ be a set of $n$-points in general position in $\mathbb{R}^{d}$. Then there is a point in at least $c_{4}(d)\left(\begin{array}{c}n \\ d+1\end{array}\right)$ d-simplices spanned by $P$.

Theorem 1.4 was proved by Boros and Füredi 4 in the plane and it was generalized to arbitrary dimension by the first author 2. Relatively recent extensive work of Gromov 9] implies a topological version of Theorem [1.4, see Theorem 4.1 for the precise statement of this extension. In addition, Gromov's approach yielded a significant improvement of the lower bound for the highest possible value of the constant $c_{4}(d)$ in Theorem 1.4.

From this point of view, it is desirable to know whether there is a topological extension of Theorem 1.1 which could also possibly be quantitatively stronger with respect to the constant $c_{1}(d)$. However, Theorem 1.2 shows that in the case of this homogeneous selection theorem we would ask for too much.

A brief proof overview. Our proof of Theorem 1.2 partially builds on the approach from [14] where the homogeneous selection theorem was used to distinguish a geometric and a topological invariant.

For the proof of Theorem 1.2 we need to exhibit a continuous map $f:\left(\Delta_{n-1}^{(0)}\right)^{*(d+1)} \rightarrow \mathbb{R}^{d}$ such that $\tau(f)$ is low, namely at most $c_{2}(d) n^{1 / d}$. Our result is in fact stronger: For some $N \geq$ $(d+1) n$, we construct a map $f: \Delta_{N-1} \rightarrow \mathbb{R}^{d}$ such that for any pairwise disjoint $n$-subsets 
$V_{1}, \ldots, V_{d+1}$ of the vertex set of $\Delta_{N-1}$, the restriction of $f$ to $V_{1} * \cdots * V_{d+1} \cong\left(\Delta_{n-1}^{(0)}\right)^{*(d+1)}$ satisfies

$$
\tau\left(f_{\mid V_{1} * \cdots * V_{d+1}}\right) \leq c_{2}(d) n^{1 / d}
$$

The construction of $f$ proceeds roughly as follows (see Sections 2 and 3 for the relevant definitions). Let $L$ be any finite graded lattice of rank $d+1$ with minimal element $\widehat{0}$, whose set of atoms $A$ satisfies $|A|=N \geq n(d+1)$. Let $S(A) \cong \Delta_{N-1}$ be the simplex on the vertex set $A$, and let $\tilde{L}=L-\{\widehat{0}\}$. We first observe (see Claim [3.2) that there exists a continuous map $g$ from $S(A)$ to the order complex $\Delta(\tilde{L})$ such that $g\left(\left\langle a_{0}, \ldots, a_{p}\right\rangle\right) \subset \Delta\left(\tilde{L}_{\leq \vee_{i=0}^{p} a_{i}}\right)$ for any atoms $a_{0}, \ldots, a_{p} \in A$ (in words: $\left\langle a_{0}, \ldots, a_{p}\right\rangle$ maps into the subcomplex below the join of the atoms $a_{0}, \ldots, a_{p} \in A$ in the order complex of $\left.\tilde{L}\right)$. Next we define $f: S(A) \rightarrow \mathbb{R}^{d}$ as the composition $e \circ g$, where $e: \Delta(\tilde{L}) \rightarrow \mathbb{R}^{d}$ is the affine extension of a generic map from $\tilde{L}$ to $\mathbb{R}^{d}$.

Our main technical result, Theorem 2.1, provides an upper bound on $\tau\left(f_{\mid V_{1} * \cdots * V_{d+1}}\right)$ in terms of the expansion of the bipartite graph $G_{L}$ of atoms vs. coatoms of $L$. The desired bound (11) follows from Theorem 2.1 by choosing $L$ to be the lattice of linear subspaces of the vector space $\mathbb{F}_{q}^{d+1}$ over the finite field with $q$ elements (for suitable $q=q(n, d)$ ), and utilizing a well known expansion property of the corresponding graph $G_{L}$.

The paper is organized as follows: In Section 2 we state Theorem 2.1 and apply it to prove Theorem 1.2. The proof of Theorem 2.1] is given in Section 3, In Section 4 we prove Theorem [1.3 as a direct application of results of Gromov [9] and Erdős [8].

Subsequent work. Considering our work, Bukh and Hubard [5] very recently improved the bound on $\tau(d, n)$ to $\tau(d, n) \leq 30(\ln n)^{1 /(d-1)}$.

\section{Finite Lattices and Topological Pach Numbers}

A finite poset $(L,<)$ is a lattice if for any two element $x, y \in L$ the set $\{z: z \leq x, z \leq y\}$ has a unique maximal element $x \wedge y$, and the set $\{z: z \geq x, z \geq y\}$ has a unique minimal element $x \vee y$. In particular, a lattice has a minimal element $\widehat{0}$ and a maximal element $\widehat{1}$. A lattice $L$ is graded with rank function $\operatorname{rk}: L \rightarrow \mathbb{N}$, if $\operatorname{rk}(\widehat{0})=0$ and if $\operatorname{rk}(y)=\operatorname{rk}(x)+1$ whenever $y$ covers $x$ (i.e. $\{z: x \leq z \leq y\}=\{x, y\}$ ). See Stanley's book [13] for a comprehensive reference on the combinatorics of posets and lattices.

Let $L$ be a graded lattice of $\operatorname{rank} \operatorname{rk}(\widehat{1})=d+1$. Let

$$
A=\{x \in L: \operatorname{rk}(x)=1\} \quad, \quad C=\{x \in L: \operatorname{rk}(x)=d\}
$$

be respectively the sets of atoms and coatoms of $L$. For $x \in L$ let

$$
A_{x}=\{a \in A: a \leq x\}, C_{x}=\{c \in C: x \leq c\} .
$$

Let $G_{L}$ denote the bipartite graph on the vertex set $A \cup C$ with edges $(a, c) \in A \times C$ iff $a \leq c$. For a set of atoms $Z \subset A$ let $\Gamma(Z)=\cup_{z \in Z} C_{z}$ be the neighborhood of $Z$.

The main ingredient of the proof of Theorem 1.2 is the following connection between $\tau(d, n)$ and the expansion of $G_{L}$. 
Theorem 2.1. Let $L$ be a graded lattice of rank $d+1$ such that $|A| \geq n(d+1)$. Then $m=\tau(d, n)$ satisfies

$$
\min _{Z \subset A,|Z|=m}|\Gamma(Z)| \leq \frac{d}{d+1}\left(\max _{a \in A}\left|C_{a}\right|+|C|\right) .
$$

The proof of Theorem 2.1 is deferred to Section 3 .

Proof of Theorem 1.2; Let $n \geq(2 d)^{d}$. By Bertrand's postulate there exists a prime $q$ such that

$$
2 d \leq((d+1) n)^{1 / d} \leq q \leq 2((d+1) n)^{1 / d} .
$$

Let $\mathbb{F}_{q}$ be the finite field of order $q$. Let $L=L(d+1, q)$ denote the graded lattice of linear subspaces of $\mathbb{F}_{q}^{d+1}$ ordered by inclusion, with the natural $\operatorname{rank}$ function $\operatorname{rk}(x)=\operatorname{dim} x$ for all $x \in L$. The sets of atoms and coatoms of $L$ satisfy $|A|=|C|=N_{d}=\frac{q^{d+1}-1}{q-1}$ and $\left|C_{a}\right|=N_{d-1}=\frac{q^{d}-1}{q-1}$ for all $a \in A$. Any two distinct 1-dimensional subspaces of $\mathbb{F}_{q}^{d+1}$ are contained in exactly $N_{d-2}=\frac{q^{d-1}-1}{q-1}$ hyperplanes of $\mathbb{F}_{q}^{d+1}$. Hence, if $a \neq a^{\prime} \in A$ are two distinct atoms then

$$
\left|C_{a} \cap C_{a^{\prime}}\right|=N_{d-2}=\frac{q^{d-1}-1}{q-1} .
$$

It follows that if $Z \subset A$, then the family $\left\{C_{a}: a \in Z\right\}$ forms an $N_{d-1}$-uniform hypergraph on vertex set $\Gamma(Z)$ with $|Z|$ edges, and any two distinct edges intersect in a set of size $N_{d-2}$. Applying a result of Corrádi [6] (see also exercise 13.13 in [10] and Theorem 2.3(ii) in [1]) we obtain the following lower bound on the expansion of $G_{L}$.

$$
\begin{aligned}
|\Gamma(Z)| & \geq \frac{|Z| N_{d-1}^{2}}{N_{d-1}+(|Z|-1) N_{d-2}}=\frac{|Z| N_{d-1}^{2}}{q^{d-1}+|Z| N_{d-2}} \\
& =N_{d}-\frac{q^{d-1}\left(N_{d}-|Z|\right)}{q^{d-1}+|Z| N_{d-2}} \geq N_{d}-\frac{q^{d-1} N_{d}}{|Z| N_{d-2}} \\
& \geq N_{d}-\frac{q N_{d}}{|Z|} \geq N_{d}-\frac{N_{d}^{1+\frac{1}{d}}}{|Z|} .
\end{aligned}
$$

Next note that (2) implies that $|A|=N_{d} \geq q^{d} \geq(d+1) n$. Applying Theorem 2.1 together with (3), it follows that $m=\tau(d, n)$ satisfies

$$
\begin{aligned}
N_{d}-\frac{N_{d}^{1+\frac{1}{d}}}{m} & \leq \min _{Z \subset A,|Z|=m}|\Gamma(Z)| \\
& \leq \frac{d}{d+1}\left(\max _{a \in A}\left|C_{a}\right|+|C|\right) \\
& =\frac{d}{d+1}\left(N_{d-1}+N_{d}\right) .
\end{aligned}
$$

The assumption $q \geq 2 d$ implies that

$$
\begin{aligned}
\frac{N_{d}}{N_{d}-d N_{d-1}} & =\frac{q^{d+1}-1}{q^{d+1}-1-d\left(q^{d}-1\right)} \\
& \leq \frac{q^{d+1}}{q^{d+1}-d q^{d}}=\frac{q}{q-d} \leq 2 .
\end{aligned}
$$


Rearranging (4) and using (5) and $q^{d} \leq 2^{d}(d+1) n$, we obtain

$$
\begin{aligned}
m & \leq \frac{(d+1) N_{d}^{1+\frac{1}{d}}}{N_{d}-d N_{d-1}} \leq 2(d+1) N_{d}^{\frac{1}{d}} \\
& \leq 2(d+1)\left((d+1) q^{d}\right)^{1 / d} \\
& \leq 2(d+1)\left((d+1)\left(2^{d}(d+1) n\right)\right)^{1 / d} \\
& =4(d+1)\left((d+1)^{2} n\right)^{1 / d} .
\end{aligned}
$$

\section{Continuous Maps of Finite Lattices}

In this section we prove Theorem 2.1, We first recall some definitions. The order complex $\Delta(P)$ of a finite poset $(P,<)$ is the simplicial complex on the vertex set $P$, whose $k$-simplices are the chains $x_{0}<\cdots<x_{k}$ in $P$.

Let $L$ be a graded lattice of $\operatorname{rank} d+1$ and let $\tilde{L}=L-\{\widehat{0}\}$. For a subset $\sigma \subset L$ let $\vee \sigma=\vee_{x \in \sigma} x$. Let $S(A)$ be the simplex on the set $A$ of atoms of $L$ (identified as usual with its geometric realization). For $x \in \tilde{L}$ let $\tilde{L}_{\leq x}=\{y \in \tilde{L}: y \leq x\}$. The main ingredient in the proof of Theorem 2.1 is the following result.

Proposition 3.1. There exists a continuous map $f: S(A) \rightarrow \mathbb{R}^{d}$ such that for any $u \in \mathbb{R}^{d}$

$$
\left|\left\{c \in C: u \in f\left(\left\langle A_{c}\right\rangle\right)\right\}\right| \leq d \max _{a \in A}\left|C_{a}\right| .
$$

(Note that, in accordance with our notation, $\left\langle A_{c}\right\rangle$ stands here for the geometric realization of $A_{c}$, considered as a face of $S(A)$.)

We first note the following

Claim 3.2. There exists a continuous map $g: S(A) \rightarrow \Delta(\tilde{L})$ such that for all $x \in \tilde{L}$

$$
g\left(\left\langle A_{x}\right\rangle\right) \subset \Delta\left(\tilde{L}_{\leq x}\right) .
$$

Proof: We define $g$ inductively on the $k$-skeleton $S(A)^{(k)}$. On the vertices $a \in A$ of $S(A)$ let $g(a)=a$. Let $0<k \leq|A|-1$ and suppose $g$ has been defined on $S(A)^{(k-1)}$. Let $\sigma=\left\langle a_{0}, \ldots, a_{k}\right\rangle \in S(A)^{(k)}$ and let $y=\vee \sigma$. For $0 \leq i \leq k$ let

$$
\sigma_{i}=\left\langle a_{0}, \ldots, a_{i-1}, \widehat{a_{i}}, a_{i+1}, \ldots, a_{k}\right\rangle
$$

be the $i$-th face of $\sigma$. Let $y_{i}=\vee \sigma_{i}$. Then $g$ is defined on $\sigma_{i}$ and by induction hypothesis

$$
g\left(\sigma_{i}\right) \subset \Delta\left(\tilde{L}_{\leq y_{i}}\right) \subset \Delta\left(\tilde{L}_{\leq y}\right) .
$$

Being a cone, $\Delta\left(\tilde{L}_{\leq y}\right)$ is contractible and hence $g$ can be continuously extended from the boundary $\partial \sigma$ to the whole of $\sigma$ so that $g(\sigma) \subset \Delta\left(\tilde{L}_{\leq y}\right)$. It follows in particular that for $x \in \tilde{L}$

$$
g\left(\left\langle A_{x}\right\rangle\right) \subset \Delta\left(\tilde{L}_{\leq \vee A_{x}}\right) \subset \Delta\left(\tilde{L}_{\leq x}\right) .
$$


Proof of Proposition 3.1; By a general position argument we choose a mapping $e$ : $\tilde{L} \rightarrow \mathbb{R}^{d}$ with the following property: For any pairwise disjoint subsets $S_{1}, \ldots, S_{d+1} \subset \tilde{L}$ of cardinalities $\left|S_{i}\right| \leq d$, it holds that

$$
\bigcap_{i=1}^{d+1} \operatorname{aff}\left(e\left(S_{i}\right)\right)=\emptyset
$$

and thus in particular

$$
\bigcap_{i=1}^{d+1} \operatorname{relint} \operatorname{conv}\left(e\left(S_{i}\right)\right)=\emptyset .
$$

Extend $e$ by linearity to the whole of $\Delta(\tilde{L})$ and let $f=e \circ g: S(A) \rightarrow \mathbb{R}^{d}$, where $g$ is the map from Claim 3.2. We claim that the map $f$ satisfies (6). Let $u \in \mathbb{R}^{d}$ and let

$$
T=\{\eta \in \Delta(\tilde{L}): u \in \operatorname{relint} e(\langle\eta\rangle)\} .
$$

Choose a maximal pairwise disjoint subfamily $T^{\prime} \subset T$. It follows by (7) that $\left|T^{\prime}\right| \leq d$. For each $\eta^{\prime} \in T^{\prime}$ choose an atom $a\left(\eta^{\prime}\right) \in A$ such that

$$
a\left(\eta^{\prime}\right) \leq \min \eta^{\prime} .
$$

Now let $c \in C$ be such that $u \in f\left(\left\langle A_{c}\right\rangle\right)$. Then there exists a $b \in g\left(\left\langle A_{c}\right\rangle\right) \subset \Delta\left(\tilde{L}_{\leq c}\right)$ such that $u=e(b)$. Let $\eta \in T$ be such that $b \in \operatorname{relint}\langle\eta\rangle$. Then

$$
\eta \in \Delta\left(\tilde{L}_{\leq c}\right) .
$$

By maximality of $T^{\prime}$ there exists a simplex $\eta^{\prime} \in T^{\prime}$ and a vertex $x \in \eta^{\prime} \cap \eta$. It follows by (8) and (9)) that $a\left(\eta^{\prime}\right) \leq x \leq c$, i.e. $c \in C_{a\left(\eta^{\prime}\right)}$ (see figure 1). Therefore

$$
\left|\left\{c \in C: u \in f\left(\left\langle A_{c}\right\rangle\right)\right\}\right| \leq \sum_{\eta^{\prime} \in T^{\prime}}\left|C_{a\left(\eta^{\prime}\right)}\right| \leq d \max _{a \in A}\left|C_{a}\right| .
$$

Proof of Theorem 2.1: Let $L$ be a lattice of rank $d+1$ whose set of atoms $A$ satisfies $|A| \geq(d+1) n$. Let $V_{1}, \ldots, V_{d+1}$ be disjoint $n$-subsets of $A$. By Proposition 3.1 there exists a continuous map $f: S(A) \rightarrow \mathbb{R}^{d}$ such that for any $u \in \mathbb{R}^{d}$

$$
\left|\left\{c \in C: u \in f\left(\left\langle A_{c}\right\rangle\right)\right\}\right| \leq d \max _{a \in A}\left|C_{a}\right| .
$$

Let $m=\tau(d, n)$. Then there exist $Z_{1} \subset V_{1}, \ldots, Z_{d+1} \subset V_{d+1}$ and a $u \in \mathbb{R}^{d}$ such that $\left|Z_{i}\right| \geq m$ for all $1 \leq i \leq d+1$ and

$$
u \in \bigcap_{z_{1} \in Z_{1}, \ldots, z_{d+1} \in Z_{d+1}} f\left(\left\langle z_{1}, \ldots, z_{d+1}\right\rangle\right)
$$

Write

$$
C\left(Z_{1}, \ldots, Z_{d+1}\right)=\bigcap_{i=1}^{d+1}\left\{c \in C: A_{c} \cap Z_{i} \neq \emptyset\right\}
$$




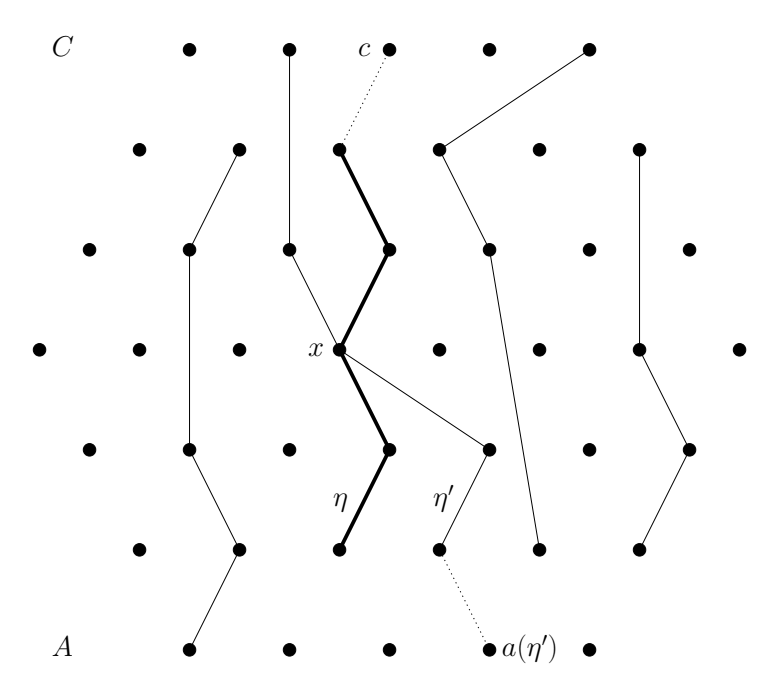

Figure 1: The bold chain corresponds to $\eta$. The other chains represent simplices of $T^{\prime}$.

If $c \in C\left(Z_{1}, \ldots, Z_{d+1}\right)$ then there exist $z_{1} \in Z_{1}, \ldots, z_{d+1} \in Z_{d+1}$ such that $z_{i} \leq c$ for all $i$ and hence $u \in f\left(\left\langle z_{1}, \ldots, z_{d+1}\right\rangle\right) \subset f\left(\left\langle A_{c}\right\rangle\right)$. Hence by Proposition 3.1

$$
\left|C\left(Z_{1}, \ldots, Z_{d+1}\right)\right| \leq d \max _{a \in A}\left|C_{a}\right| .
$$

On the other hand

$$
\begin{aligned}
\left|C\left(Z_{1}, \ldots, Z_{d+1}\right)\right| & =\left|C-\bigcup_{i=1}^{d+1}\left(C-\Gamma\left(Z_{i}\right)\right)\right| \\
& \geq|C|-\sum_{i=1}^{d+1}\left(|C|-\left|\Gamma\left(Z_{i}\right)\right|\right)=\sum_{i=1}^{d+1}\left|\Gamma\left(Z_{i}\right)\right|-d|C| \\
& \geq(d+1) \min _{Z \subset A,|Z|=m}|\Gamma(Z)|-d|C| .
\end{aligned}
$$

Theorem 2.1 now follows from (10) and (11).

Remark: The mapping $g: S(A) \rightarrow \Delta(\tilde{L})$ constructed in Claim 3.2 is in general not simplicial. It follows (as of course must be the case by Theorem 1.1) that $f=e \circ g: S(A) \rightarrow$ $\mathbb{R}^{d}$ is not affine.

\section{The Lower Bound}

Theorem 1.3 is a direct consequence of Gromov's topological overlap Theorem [9] combined with a result of Erdős on complete $(d+1)$-partite subhypergraphs in $(d+1)$-uniform dense hypergraphs [8]. We first recall these results. Let $X$ be a finite $d$-dimensional pure simplicial complex. For $k \geq 0$, let $f_{k}(X)=|X(k)|$ denote the number of $k$-dimensional faces of $X$. 
Define a positive weight function $w=w_{X}$ on the simplices of $X$ as follows. For $\sigma \in X(k)$, let $c(\sigma)=|\{\eta \in X(d): \sigma \subset \eta\}|$ and let

$$
w(\sigma)=\frac{c(\sigma)}{\left(\begin{array}{l}
d+1 \\
k+1
\end{array}\right) f_{d}(X)} .
$$

Let $C^{k}(X)$ denote the space of $\mathbb{F}_{2}$-valued $k$-cochains of $X$ with the coboundary map $d_{k}$ : $C^{k}(X) \rightarrow C^{k+1}(X)$. As usual, the space of $k$-coboundaries is denoted by $d_{k-1}\left(C^{k-1}(X)\right)=$ $B^{k}(X)$. For $\phi \in C^{k}(X)$, let $[\phi]$ denote the image of $\phi$ in $C^{k}(X) / B^{k}(X)$. Let

$$
\|\phi\|=\sum_{\sigma \in X(k): \phi(\sigma) \neq 0} w(\sigma)
$$

and

$$
\|[\phi]\|=\min \left\{\left\|\phi+d_{k-1} \psi\right\|: \psi \in C^{k-1}(X)\right\} .
$$

The $k$-th coboundary expansion constant of $X$ is

$$
h_{k}(X)=\min \left\{\frac{\left\|d_{k} \phi\right\|}{\|[\phi]\|}: \phi \in C^{k}(X)-B^{k}(X)\right\} .
$$

Note that $h_{k}(X)=0$ iff $\tilde{H}^{k}\left(X ; \mathbb{F}_{2}\right) \neq 0$. One may regard $h_{k}(X)$ as a sort of distance between $X$ and the family of complexes $Y$ that satisfy $\tilde{H}^{k}\left(Y ; \mathbb{F}_{2}\right) \neq 0$. Gromov's celebrated topological overlap result is the following:

Theorem 4.1 (Gromov [9]). For any integer $d \geq 0$ and any $\epsilon>0$ there exists a $\delta=\delta(d, \epsilon)>$ 0 such that if $h_{k}(X) \geq \epsilon$ for all $0 \leq k \leq d-1$, then for any continuous map $f: X \rightarrow \mathbb{R}^{d}$ there exists a point $u \in \mathbb{R}^{d}$ such that

$$
|\{\sigma \in X(d): u \in f(\sigma)\}| \geq \delta f_{d}(X) .
$$

We next describe a result of Erdős that generalizes the well known Erdős-Stone and Kövári-Sós-Turán theorems from graphs to hypergraphs.

Theorem 4.2 (Erdös [8]). For any $d$ and $c^{\prime}>0$ there exists a constant $c=c\left(d, c^{\prime}\right)>0$ such that for any $(d+1)$-uniform hypergraph $\mathcal{F}$ on $N$-element set $V$ with at least $c^{\prime} N^{d+1}$ hyperedges, there exists an $m \geq c(\log N)^{1 / d}$ and disjoint m-element sets $Z_{1}, \ldots, Z_{d+1} \subset V$ such that $\left\{z_{1}, \ldots, z_{d+1}\right\} \in \mathcal{F}$ for all $z_{1} \in Z_{1}, \ldots, z_{d+1} \in Z_{d+1}$.

Proof of Theorem 1.3; Recall that $V_{1}, \ldots, V_{d+1}$ are disjoint $n$-element sets and let $V=$ $V_{1} \cup \cdots \cup V_{d+1},|V|=N=(d+1) n$. Let $X=V_{1} * \ldots * V_{d+1}$ and let $f: X \rightarrow \mathbb{R}^{d}$ be a continuous map. It was shown by Gromov [9] (see also [7, 11]) that the expansion constants $h_{i}(X)$ are uniformly bounded away from zero. Concretely, it follows from Theorem 3.3 in [11] that $h_{i}(X) \geq \epsilon=2^{-d}$ for $0 \leq i \leq d-1$. Let $\delta=\delta\left(d, 2^{-d}\right)$. Then by Theorem 4.1 there exists a $u \in \mathbb{R}^{d}$ and a family $\mathcal{F} \subset X(d)$ of cardinality

$$
|\mathcal{F}| \geq \delta f_{d}(X)=\delta n^{d+1}=\delta(d+1)^{-(d+1)} N^{d+1}
$$

such that $u \in f(\sigma)$ for all $\sigma \in \mathcal{F}$. Writing $c^{\prime}=\delta(d+1)^{-(d+1)}$ and $c_{3}(d)=c\left(d, c^{\prime}\right)$, it follows from Theorem 4.2 that there exists an $m \geq c_{3}(d)(\log N)^{1 / d} \geq c_{3}(d)(\log n)^{1 / d}$ and disjoint $m$-sets $Z_{1}, \cdots, Z_{d+1} \subset V$ such that $u \in f\left(\left\langle z_{1}, \ldots, z_{d+1}\right\rangle\right)$ for all $z_{1} \in Z_{1}, \ldots, z_{d+1} \in Z_{d+1}$. Clearly, there exists a permutation $\pi$ on $\{1, \ldots, d+1\}$ such that $Z_{\pi(i)} \subset V_{i}$ for all $1 \leq i \leq d+1$. 


\section{Acknowledgements}

This research was supported by ERC Advanced Research Grant no 267165 (DISCONV). Imre Bárány is partially supported by Hungarian National Research Grant K 111827. Roy Meshulam is partially supported by ISF grant 326/16 and GIF grant 1261/14, Eran Nevo by ISF grant 1695/15 and Martin Tancer by GAČR grant 16-01602Y.

\section{References}

[1] Alon, N.: Eigenvalues, geometric expanders, sorting in rounds, and Ramsey theory. Combinatorica 6, 207-219 (1986)

[2] Bárány, I.: A generalization of Carathéodory's theorem. Discrete Math. 40, 141-152 (1982)

[3] Bárány, I., Füredi, Lovász, L.: On the number of halving planes. Combinatorica 10, 175-183 (1990)

[4] Boros, E., Füredi, Z.: The number of triangles covering the center of an $n$-set. Geom. Dedicata 17, 69-77 (1984)

[5] Bukh, B., Hubard, A.: On a topological version of Pach's overlap theorem, arXiv:1708.04350.

[6] Corrádi, K.: Problem at the Schweitzer Competition. Mat. Lapok 20, 159-162 (1969)

[7] Dotterrer, D., Kahle, M.: Coboundary expanders. J. Topol. Anal. 4, 499-514 (2012)

[8] Erdős, P.: On extremal problems of graphs and generalized graphs. Israel J. Math. 2, 183-190 (1964)

[9] Gromov, M.: Singularities, expanders and topology of maps. Part 2: From combinatorics to topology via algebraic isoperimetry. Geom. Funct. Anal. 20, 416-526 (2010)

[10] Lovász, L.: Combinatorial problems and exercises. Second edition. North-Holland Publishing Co., Amsterdam (1993)

[11] Lubotzky, A., Meshulam, R., Mozes, S.: Expansion of building-like complexes. Groups Geom. Dyn. 10, 155-175 (2016)

[12] Pach, J.: A Tverberg-type result on multicolored simplices. Comput. Geom.: Theor. Appl. 10, 71-76 (1998)

[13] Stanley, R. P.: Enumerative combinatorics. Volume 1. Second edition. Cambridge Studies in Advanced Mathematics, 49. Cambridge University Press, Cambridge (2012)

[14] Tancer, M.: Non-representability of finite projective planes by convex sets. Proc. Amer. Math. Soc. 138, 3285-3291 (2010) 